\title{
Rehearsal and modality effects on delayed recall of continuously presented
} paired associates*

\author{
DONNA L. BREITENSTEIN† \\ College of William and Mary, Williamsburg, Va. 23185
}

Number-letter pairs were presented either visually or aurally in a continuous sequence with intervals of written or spoken rehearsal. Modality effects were found only in presentation method, with no difference between written and spoken recall. Even though attention to stimulus items was assured by both written and oral rehearsal, only oral rehearsal facilitated recall. Recall was best when items were presented aurally and rehearsed orally. Therefore, oral rehearsal facilitates memory directly and is not an artifact of increased attention.

Rehearsal by vocalizing improves memory (Brelsford \& Atkinson, 1968; Murray, 1965, 1966). This improved recall may occur because $S$ attends more to stimuli he must rehearse or because the vocalization may affect the probability of the stimuli's entering a rehearsal buffer (Brelsford \& Atkinson, 1968). Writing the stimulus would also require $S$ to attend to it. The present experiment compares recall of paired associates after written rehearsal to recall after oral rehearsal. In an attempt to limit rehearsal to the type being studied, $S$ performed either an unrelated written task while he was rehearsing orally or an oral task during the written rehearsal. An additional group of $\mathrm{Ss}$ performed two nonrehearsal tasks to limit both types of overt rehearsal.

Since modality affects recall (Craik, 1969, 1970; Murdock, 1966, 1967), stimuli were presented either visually or aurally. The modality of $S$ responding was also varied. The continuous presentation method (Brelsford \& Atkinson, 1968) was used to provide experimentally controlled interference between presentation and test.

\section{SUBJECTS}

Sixty introductory psychology students, 30 males and 30 females, volunteered to serve as Ss.

APPARATUS

For aural presentation, the stimuli were recorded and played by a tape recorder. For visual presentation, the stimuli were filmed on $16-\mathrm{mm}$ movie film and projected by a Perceptoscope (Model 5102-2, Perceptual Development Laboratories).

*This paper was adapted from a thesis submitted to the college of William and Mary in partial fulfillment of the requirements of the degree of Master of Arts. The author gratefully acknowledges the guidance of Peter $L$. Derks and the valuable comments of Herbert Friedman, Ben A. Hammack, and E. Rae Harcum.

+ Now at Eastern State Hospital, Williamsburg, Va. 23185.

\section{STIMULI AND RESPONSES}

Stimuli were the four two-digit numbers: $33,44,55$, and 66 . Responses were the letters $\mathrm{K}, \mathrm{L}, \mathrm{N}, \mathrm{O}$, $\mathrm{R}, \mathrm{S}, \mathrm{T}, \mathrm{U}, \mathrm{Y}$, which were chosen because they neither looked nor sounded similar to one another. Each response was paired with each stimulus about an equal number of times. Two lists, each of 200 trials, were constructed with six intervening trials, the greatest lag between presentation of a pair and the test. The Ss were instructed to remember the most recent response paired with each stimulus.

\section{PROCEDURE}

Initially, four pairs were presented for study, in this order: (1) the word "study" for 1 sec, (2) S-R pair, (3) $3-\mathrm{sec}$ interval for rehearsal. Steps 1-3 were repeated three times, so each of the four stimuli was introduced with a response. Then trials began, consisting of: (4) the word "test" for $1 \mathrm{sec}$, (5) stimulus probe (one of the two-digit numbers), (6) 3-sec interval for $S$ to recall the response to the stimulus presented, (1) the word "study" for $1 \mathrm{sec}$, (2) S-R pair, of which the stimulus was the one just tested in Step 5 , with a new response, (3) 3-sec interval for rehearsal. The sequence was then repeated for the second trial. The Ss were given a practice list with 10 complete trials, then the two experimental lists.

The Ss were tested individually on one of six conditions. Each $S$ received lists in one modality, either aural or visual, and performed in one of three rehearsal conditions. For oral rehearsal (OR) Ss said aloud the S-R pair and repeated it continuously for the entire interval. While verbalizing the S-R pair, these Ss wrote digits beginning $1,2,3$, etc. For written rehearsal (WR), Ss wrote down the $S-R$ pair and continued writing it during the entire rehearsal interval. While writing, these Ss counted digits aloud. In the condition with no overt rehearsal (NOR), Ss performed both an oral task (saying the alphabet) and a written task (writing digits). Each $S$ was tested on two lists-one with visual stimulus probe and written recall, and the other with oral probe and spoken recall. Half of the Ss were given the visual probe condition first.

\section{RESULTS AND DISCUSSION}

The percentage of correct responses at each lag of recall was computed for each S. A split-plot analysis of variance was performed for the variables of presentation mode, recall mode, method of rehearsal, and lag ( 2 by 2 by 3 by 7 ), with $10 \mathrm{Ss}$ in each condition. Significant main effects were found for presentation method $[F(1,378)=30.25, \quad p<.001]$, rehearsal method $[F(2,378)=8.15$, $\mathrm{p}<.001]$, and recall $\operatorname{lag}[\mathrm{F}(6,378)=$ $297.56, p<.001$ ]. The only significant interaction was a triple interaction of presentation method, recall method, and lag $[F(6,378)=$ $2.30, \mathrm{p}<.05]$. Figure 1 shows the percentage of correct recalls at each lag for presentation and rehearsal conditions.

Recall decreased as the lag increased; the greatest loss of information was between Lags 0 and 1. Recall in all groups greatly decreased with increasing lags in similarly shaped curves. After six intervening trials, mean performance had not quite reached the chance level of guessing one correct out of the nine possible responses $(11.11 \%)$.

For each rehearsal condition, aural presentation resulted in better recall than visual presentation at every lag. Mode of output had no effect except in interaction with presentation

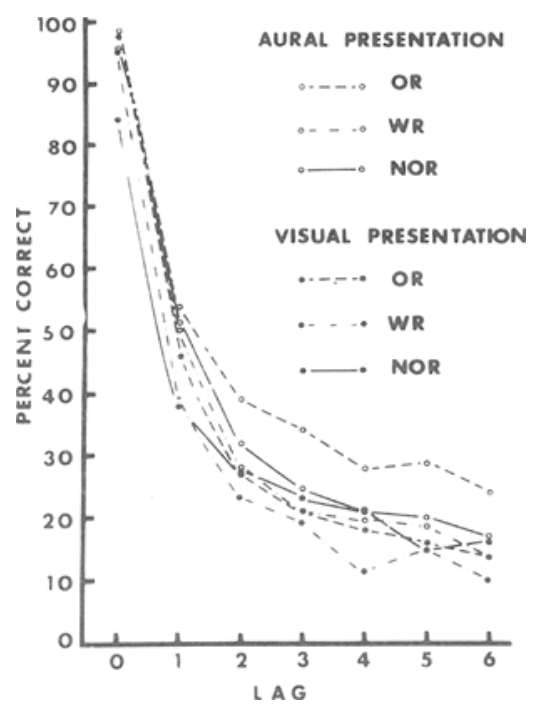

Fig. 1. Percentage of correct responses as a function of lag for each presentation and rehearsal condition. 
method. Spoken recall of aurally presented items was significantly better than spoken recall of visually presented items at every lag except a lag of zero, where differences were not significant. Written recall of aurally presented items was significantly better than with visually presented items for all lags except 3 and 6 , where there were no significant differences. Thus, mode of output had little effect on recall; instead, the mode of presentation caused the modality differences.

Recall was significantly better after OR than after WR or NOR. After a lag of six, performance after WR was not significantly different from chance $[t(39)=1.00]$. Even with restricted rehearsal, $S$ was able to retain information; performance after NOR approached chance, but it was still significantly different $[t(39)=2.52$, $\mathrm{p}<.05]$. Performance after OR was significantly greater than chance $[\mathrm{t}(39)=3.71, \mathrm{p}<.001]$ at $\operatorname{Lag} 6$.
Even though Ss overtly rehearsed with WR, recall was not better than after NOR. This may, in part, have been due to $S$ 's rehearsing during the recall interval; if $\mathbf{S}$ answered immediately, he could have used the remaining seconds to rehearse covertly. The number of times Ss rehearsed the pair during the rehearsal intervals varied among $S s$, and no consistent trends were found. Since both WR and OR insured that the items were attended to, the superior recall after $O R$ could not have been due to differences in attention. Instead, the increased recall after $O R$ must have resulted from a modality effect in which oral activity enhanced memory; OR may have increased the chances of an item's being stored in memory, or it may have strengthened an item in an auditory store. Since written rehearsal did not increase recall, the attention insured by overt rehearsal was not the critical variable. Modality, rather than attention, caused the differences in recall.
BRELSFORD, J. W., JR., \& ATKINSON, R . C. Recall of paired-associates as a function of overt and covert rehearsal procedures. Journal of Verbal Learning \& Verbal Behavior, 1968, 7, 730-736.

CRAIK, F. I. M. Modality effects in short-term storage. Journal of Verbal Learning \& Verbal Behavior, 1969,8, 658-664.

CRAIK, F. I. M. The fate of primary memory items in free recall. Journal of Verbal Learning \& Verbal Behavior, 1970, 9. 143-148.

MURDOCK, B. B., JR. Visual and auditory stores in short-term memory. Quarterly Journal of Experimental Psychology, 1966, 18, 206-211.

MURDOCK, B. B., JR. Auditory and visual stores in short-term memory. Acta Psychologica, 1967, 27, 316-324.

MURRAY D. J Vocalization-at-presentation and immediate recall, with varying presentation rates. Quarterly Journal of Experimental Psychology, 1965, 17, 47-56.

MURRAY, D. J. Vocalization-at-presentation and immediate recall, with varying recall methods. Quarterly Journal of Experimental Psychology, 1966, 18, 9-18. 